\title{
Zero Temperature Limits of Gibbs-Equilibrium States for Countable Alphabet Subshifts of Finite Type
}

\author{
O. Jenkinson, ${ }^{1}$ R. D. Mauldin, ${ }^{2}$ and M. Urbański ${ }^{2}$
}

Received October 14 2004; accepted January 25, 2005

\begin{abstract}
Let $\Sigma_{A}$ be a finitely primitive subshift of finite type on a countable alphabet. For appropriate functions $f: \Sigma_{A} \rightarrow I R$, the family of Gibbs-equilibrium states $\left(\mu_{t f}\right)_{t \geqslant 1}$ for the functions $t f$ is shown to be tight. Any weak*-accumulation point as $t \rightarrow \infty$ is shown to be a maximizing measure for $f$.
\end{abstract}

KEY WORDS: Gibbs state; equilibrium state; ground state; maximizing measure; countable alphabet subshift of finite type.

\section{INTRODUCTION}

Let $\Sigma_{A}$ be a subshift of finite type on a countably infinite alphabet, and suppose that the function $f: \Sigma_{A} \rightarrow I R$ has summable variations. Further assumptions on $f$ ensure it has a unique Gibbs-equilibrium state $\mu_{f}$ (see Section 2 for more details). The purpose of this article is to analyse the behaviour, as $t \rightarrow \infty$, of the Gibbs-equilibrium states $\mu_{t f}$ of $t f$. It will be shown that the family $\left(\mu_{t f}\right)_{t} \geqslant 1$ is tight, thereby ensuring that it has a weak* accumulation point. Any such accumulation point is shown to be a maximizing measure for the function $f$ (i.e. its $f$-integral dominates the integral of $f$ with respect to other shift-invariant probability measures). This extends the analogous results of refs. 7-9 which were proved in the setting of finite alphabet subshifts of finite type (see also ref. 1 for related results in the context of quantum and classical lattice models).

The thermodynamic interpretation (cf. ref. 17) of the parameter $t$ is as an inverse temperature of a system, while the measure $\mu_{t f}$ describes the

\footnotetext{
${ }^{1}$ School of Mathematical Sciences, Queen Mary, University of London, Mile End Road, London E1 4NS, UK; email: omj@maths.qmul.ac.uk

${ }^{2}$ Department of Mathematics, University of North Texas, P. O. Box 311430, Denton, TX 76203-1430, USA; email: mauldin@unt.edu, urbanski@unt.edu
} 
equilibrium of the system at temperature $1 / t$ (i.e. the one which minimizes the "free energy"). The $t \rightarrow \infty$ limit is therefore a zero temperature limit, and an accumulation point of the $\mu_{t f}$ can be interpreted as a ground state.

If $f$ has a unique maximizing measure then our result asserts that $\mu_{t f}$ will converge to that measure. A more intriguing sitution arises when there are several maximizing measures, in which case we only know that $\mu_{t f}$ will accumulate on some non-empty subset of such measures. However, in all known examples it has been observed that the family $\mu_{t f}$ does converge, so a natural conjecture is that this is always the case; if this conjecture is true then the limit of the $\mu_{t f}$ may be regarded as the most "physically relevant" maximizing measure. This problem is open even for finite alphabet subshifts of finite type, though Brémont ${ }^{(4)}$ has shown that if $f$ depends on only finitely many coordinates then the $\mu_{t f}$ do converge (cf. refs. 6, 9, 13 for related results).

We remark that Radin (see e.g. refs. 14-16) and Schrader ${ }^{(20)}$ have considered similar problems for locally constant functions defined on multidimensional full shifts $A^{\mathbb{Z}^{d}}$ where the alphabet $A$ is finite, and there is related work of Kerimov (see e.g. ref. 11) in the context of one-dimensional full shifts $A^{\mathbb{Z}}$ with countably infinite alphabet $A$, though here the ground state is always unique.

\section{PRELIMINARIES}

Let $\Sigma=I N^{I N}$ denote the full shift on the countable alphabet $I N=$ $\{1,2, \ldots\}$, equipped with the product topology.

Given an adjacency matrix $A: I N \times I N \rightarrow\{0,1\}$, let $\Sigma_{A}$ denote the associated subshift of finite type

$$
\Sigma_{A}=\left\{x \in \Sigma: A\left(x_{n}, x_{n+1}\right)=1 \text { for all } n \geqslant 1\right\} .
$$

The subshift of finite type $\Sigma_{A}$ is compact if and only if $I N_{A}:=\{i \in I N:[i] \neq$ $\emptyset\}$ is finite.

We shall suppose that $A$ is finitely primitive, i.e. there exists an integer $N \geqslant 0$, and a finite sub-alphabet $\mathbb{M} \subset I N$, such that for all $x \in \Sigma_{A}$ and all $i \in \mathbb{N}_{A}$ there exists $w \in \mathbb{M}^{N}$ with $i w x \in \Sigma_{A}$. This implies that the shift map $T: \Sigma_{A} \rightarrow \Sigma_{A}$, defined by $(T x)_{n}=x_{n+1}$, is topologically mixing.

For $n \in \mathbb{I N}$, define $\Pi_{n}: \Sigma_{A} \rightarrow \mathbb{I N}^{n}$ by $\Pi_{n}(x)=\left(x_{1}, \ldots, x_{n}\right)$, and $\pi_{n}: \Sigma_{A} \rightarrow I N$ by $\pi_{n}(x)=x_{n}$. If $w \in I^{n}$ then the corresponding cylinder set in $\Sigma_{A}$ is defined by $[w]=\left\{x \in \Sigma_{A}: \Pi_{n}(x)=w\right\}$. 
We shall assume that $f: \Sigma_{A} \rightarrow I R$ has summable variations, i.e. that

$$
V(f):=\sum_{n=1}^{\infty} \operatorname{var}_{n}(f)<\infty
$$

where

$$
\operatorname{var}_{n}(f)=\sup _{\Pi_{n}(x)=\Pi_{n}(y)}|f(x)-f(y)| .
$$

In particular this implies that $f$ is uniformly continuous (though not necessarily bounded, since $\operatorname{var}_{0}(f)=\sup _{x, y \in \Sigma_{A}}|f(x)-f(y)|$ is not included in the above sum).

We also assume that

$$
\sum_{i \in I N} \exp \left(\left.\sup f\right|_{[i]}\right)<\infty
$$

so in particular $f$ is bounded above, and is unbounded below if and only if $I N_{A}$ is infinite. The summability condition (2) allows much of the thermodynamic formalism (cf. refs. 13, 17) for finite alphabet subshifts of finite type to be generalized to the non-compact setting. ${ }^{3}$ In particular it is equivalent (ref. 12, Prop. 2.1.9) to the finiteness of the topological pressure

$$
P(f)=\lim _{n \rightarrow \infty} \frac{1}{n} \log \sum_{T^{n} y=y} \exp \left(\sup _{x \in\left[\Pi_{n}(y)\right]} \sum_{i=0}^{n-1} f\left(T^{i} x\right)\right),
$$

and implies the variational characterization (ref. 12, Thm. 2.1.8)

$$
P(f)=\sup \left\{h(m)+\int f d m: m \in \mathcal{M}, \int f d m>-\infty\right\},
$$

where $\mathcal{M}$ denotes the set of $T$-invariant Borel probability measures on $\Sigma_{A}$, and $h(m)$ the metric entropy of $m \in \mathcal{M}$.

${ }^{3}$ Our reference to this generalized thermodynamic formalism is ref. 12 (though see also ref. $18,19)$, in which $f$ is assumed to be locally Hölder (i.e. $\operatorname{var}_{n}(f) \rightarrow 0$ exponentially fast). The proofs in ref. 12 can, however, be easily adapted to the more general case where $f$ has summable variations. 
Moreover, there is (see ref. 12, Theorems 2.2.4 and 2.3.3) a unique measure $\mu_{f} \in \mathcal{M}$ for which there exist constants $C_{2}>C_{1}>0$ such that

$$
C_{1} \leqslant \frac{\mu_{f}\left[\Pi_{n}(x)\right]}{\exp \left(\sum_{i=0}^{n-1} f\left(T^{i} x\right)-n P(f)\right)} \leqslant C_{2}
$$

for all $x \in \Sigma_{A}, n \geqslant 1$. In fact (ref. 12, Thm. 2.2.7) we may choose

$$
C_{2}=\exp (4 V(f)) .
$$

The measure $\mu_{f}$ is called the Gibbs state for $f$.

Suppose furthermore that ${ }^{4}$

$$
\sum_{i \in I N} \inf \left(-\left.f\right|_{[i]}\right) \exp \left(\left.\inf f\right|_{[i]}\right)<\infty
$$

so that in particular $\int f d \mu_{f}>-\infty$.

In this case (see ref. 12, Lem. 2.2.8, Thm. 2.2.9) $\mu_{f}$ is an equilibrium state for $f$, in the sense that

$$
P(f)=h\left(\mu_{f}\right)+\int f d \mu_{f} .
$$

Indeed it is the unique equilibrium state $^{5}$ for $f$ : if $m \in \mathcal{M} \backslash\left\{\mu_{f}\right\}$ is any other invariant measure with $\int f d m>-\infty$, then $h(m)+\int f d m<P(f)$. Since $\mu_{f}$ is both the unique Gibbs state and the unique equilibrium state for $f$, we shall henceforth refer to it as the Gibbs-equilibrium state for $f$.

Since $f$ satisfies (1), (2) and (5), so does the function $t f$ for any $t \geqslant 1$. It follows that each such $t f$ also has a unique Gibbs-equilibrium state $\mu_{t f}$.

A maximizing measure for $f$ is a measure $\mu \in \mathcal{M}$ such that $\int f d \mu \geqslant$ $\int f d m$ for all $m \in \mathcal{M}$. Our assumptions on $f$ ensure (see ref. 10) that this definition of a maximizing measure is equivalent to requiring that

$$
\int f d \mu=\sup _{x \in \Sigma_{A}} \limsup _{n \rightarrow \infty} \frac{1}{n} \sum_{i=0}^{n-1} f\left(T^{i} x\right) .
$$

\footnotetext{
${ }^{4}$ Note that the lefthand side of (5) is well-defined: (2), together with the fact that $\operatorname{var}_{1}(f)<$ $\infty$, implies that $\inf \left(-\left.f\right|_{[i]}\right) \rightarrow \infty$, so that $\inf \left(-\left.f\right|_{[i]}\right)$ is positive except for finitely many $i$.

${ }^{5}$ This existence and uniqueness is proved in ref. 12 under the hypothesis of finite irreducibility (see ref. 12 for the definition), which is weaker than finite primitivity. Uniqueness also follows from ref. 5, where it is shown that if $T$ is topologically transitive (a weaker condition than finite irreducibility) then $f$ has at most one equilibrium state.
} 
They also ensure (see ref. 10) the existence of a maximizing measure. The set of maximizing measures for $f$, which in general is not a singleton, will be denoted $\mathcal{M}_{\max }(f)$. The general properties of a maximizing measure are rather different from those of a Gibbs-equilibrium state. For example the support of a Gibbs-equilibrium state is always the full space $\Sigma_{A}$, whereas a maximizing measure has full support only in the trivial situation where $f$ is cohomologous to a constant (i.e. $f=c+\varphi \circ T-\varphi$ for some $c \in I R$ and some bounded continuous $\varphi$ ). This latter fact is because if $f$ is as above then there exists a bounded continuous $\varphi$ such that the set of maxima of $f+\varphi-\varphi \circ T$ contains the support of a $T$-invariant measure (see ref. 10).

\section{PROOFS OF RESULTS}

To prove our main result, Theorem 1, we first require two preparatory lemmas. For the first of these we only use the fact that $f$ is continuous and bounded above.

Lemma 1. The map

$$
\begin{aligned}
& \mathcal{M} \longrightarrow I R \\
& \mu \longmapsto \int f d \mu
\end{aligned}
$$

is upper semi-continuous with respect to the weak* topology on $\mathcal{M}$.

Proof. Suppose that $\mu_{i} \rightarrow \mu$ in the weak* topology. That is, $\int g d \mu_{i} \rightarrow$ $\int g d \mu$ for all bounded continuous functions $g$. We must prove that

$$
\limsup _{i \rightarrow \infty} \int f d \mu_{i} \leqslant \int f d \mu
$$

Let $f_{k} \searrow f$ be a sequence of bounded continuous functions converging pointwise to $f$, for example $f_{k}=\max (f,-k)$. If $I \in I R$ is such that $I>\int f d \mu$ then $\int f_{k} d \mu<I$ for all sufficiently large $k \geqslant 1$, by the monotone convergence theorem. Choose one such $k$, and let $\delta>0$ be arbitrary. Since $f_{k}$ is a bounded continuous function, and $\mu_{i} \rightarrow \mu$ in the weak* topology, $\int f_{k} d \mu>\int f_{k} d \mu_{i}-\delta$ for all $i$ sufficiently large. But $\int f_{k} d \mu_{i} \geqslant \int f d \mu_{i}$ since $f_{k} \geqslant f$, hence

$$
I>\int f_{k} d \mu>\int f_{k} d \mu_{i}-\delta \geqslant \int f d \mu_{i}-\delta
$$


for all $i$ sufficiently large. But $\delta>0$ and $I>\int f d \mu$ were arbitrary, so in fact

$$
\int f d \mu \geqslant \int f d \mu_{i}
$$

for all $i$ sufficiently large, and (7) follows. states.

For the second lemma we only use the fact that the $\mu_{t f}$ are Gibbs

Lemma 2. The family of Gibbs-equilibrium states $\left(\mu_{t f}\right)_{t} \geqslant 1$ is tight, i.e. for all $\varepsilon>0$ there exists a compact set $K \subset \Sigma_{A}$ such that $\mu_{t f}(K)>1-\varepsilon$ for all $t \geqslant 1$.

Proof. Given $\varepsilon>0$, we will find an increasing sequence $\left(n_{k}\right)$ in $I N$ such that the compact set $K=\left\{x \in \Sigma_{A}: 1 \leqslant x_{k} \leqslant n_{k} \forall k \in I N\right\}$ satisfies $\mu_{t f}(K)>1-\varepsilon$ for all $t \geqslant 1$. Now

$$
\begin{aligned}
\mu_{t f}(K) & =\mu_{t f}\left(\Sigma_{A} \backslash \cup_{k=1}^{\infty}\left\{x \in \Sigma_{A}: x_{k}>n_{k}\right\}\right) \\
& \geqslant 1-\sum_{k=1}^{\infty} \mu_{t f}\left(\left\{x \in \Sigma_{A}: x_{k}>n_{k}\right\}\right) \\
& =1-\sum_{k=1}^{\infty} \sum_{i=n_{k}+1}^{\infty} \mu_{t f}\left(\pi_{k}^{-1}(i)\right) \\
& =1-\sum_{k=1}^{\infty} \sum_{i=n_{k}+1}^{\infty} \mu_{t f}[i],
\end{aligned}
$$

so to ensure that $\mu_{t f}(K)>1-\varepsilon$ it suffices to choose the integers $n_{k}$ such that

$$
\sum_{i=n_{k}+1}^{\infty} \mu_{t f}[i]<\frac{\varepsilon}{2^{k}} \quad \text { for all } k \in \mathbb{I N}, t \geqslant 1
$$

We now show that such a choice is possible. First, the Gibbs property (4), with $n=1$ and $f$ replaced by $t f$, gives

$$
\mu_{t f}[i] \leqslant e^{4 t V(f)} \exp \left(\sup \left\{\left.t f\right|_{[i]}\right\}-P(t f)\right) .
$$


Now let $m \in \mathcal{M}$ be any measure for which $I:=\int f d m$ is finite (e.g. we may take $m$ to be supported on a periodic orbit). From (3) we have

$$
P(t f)-t I=P(t(f-I)) \geqslant \int t(f-I) d m+h(m) \geqslant 0
$$

so together with (10) we deduce that

$$
\begin{aligned}
\mu_{t f}[i]_{A} & \leqslant e^{4 t V(f)} \exp \left(\sup \left\{\left.t(f-I)\right|_{[i]}\right\}\right) e^{-P(t(f-I))} \\
& \leqslant e^{4 t V(f)} \exp \left(\sup \left\{\left.t(f-I)\right|_{[i]}\right\}\right) \\
& =\exp \left(t\left(4 V(f)-I+\sup \left\{\left.f\right|_{[i]}\right\}\right)\right) .
\end{aligned}
$$

The summability condition (2) implies that $\left.\sup f\right|_{[i]} \rightarrow-\infty$ as $i \rightarrow \infty$, with the convention that $\left.f\right|_{[i]}=-\infty$ if $[i]=\emptyset$. In particular there exists $J \in$ $I N$ such that if $i \geqslant J$ then

$$
4 V(f)-I+\left.\sup f\right|_{[i]}<0 .
$$

So, if $t \geqslant 1$ and $i \geqslant J$ then $t\left(4 V(f)-I+\left.\sup f\right|_{[i]}\right)<4 V(f)-I+\left.\sup f\right|_{[i]}<$ 0 , and from (11) we obtain

$$
\mu_{t f}[i] \leqslant e^{4 V(f)-I} e^{\left.\sup f\right|_{[i]}} .
$$

The summability condition (2) means there exists $n_{k} \geqslant J$ such that

$$
\sum_{i=n_{k}+1}^{\infty} e^{\left.\sup f\right|_{[i]}}<\frac{\varepsilon}{2^{k}} e^{I-4 V(f)},
$$

and combined with (12) we deduce (9), as required.

Theorem 1. The family of Gibbs measures $\left(\mu_{t f}\right)_{t} \geqslant 1$ has a weak* accumulation point as $t \rightarrow \infty$. Any such accumulation point $\mu$ is a maximizing measure for $f$, and $\int f d \mu=\lim _{t \rightarrow \infty} \int f d \mu_{t f}$.

Proof. By Lemma 2 the family $\left(\mu_{t f}\right)_{t} \geqslant 1$ is tight, so by Prohorov's theorem ref. 2 , p. 37 there exists at least one weak* accumulation point.

Now suppose $\mu$ is any such accumulation point. If $p(t)=P(t f)$ for $t \geqslant 1$ then $p$ is real analytic (cf. ref. 12, Thm. 2.6.12), and $p^{\prime}(t)=\int f d \mu_{t f}$ (cf. ref. 12, Prop. 2.6.13). But (3) implies that $p$ is convex, so that $t \mapsto$ $p^{\prime}(t)=\int f d \mu_{t f}$ is non-decreasing, and bounded above by $\sup f$. It follows 
that the $\operatorname{limit}_{\lim _{t \rightarrow \infty}} p^{\prime}(t)=\lim _{t \rightarrow \infty} \int f d \mu_{t f}$ exists and is finite. Moreover, Lemma 1 gives

$$
\lim _{t \rightarrow \infty} \int f d \mu_{t f} \leqslant \int f d \mu .
$$

In particular $\int f d \mu>-\infty$. From (3) and (6) it follows that

$$
\int t f d \mu_{t f}+h\left(\mu_{t f}\right) \geqslant \int t f d \mu+h(\mu),
$$

so

$$
\int f d \mu_{t f}+\frac{h\left(\mu_{t f}\right)}{t} \geqslant \int f d \mu+\frac{h(\mu)}{t} .
$$

Now $h\left(\mu_{t f}\right)=P(t f)-t \int f d \mu_{t f}=p(t)-t p^{\prime}(t)$, so

$$
\frac{d}{d t} h\left(\mu_{t f}\right)=-t p^{\prime \prime}(t)<0
$$

for $t \geqslant 1$. Therefore $h\left(\mu_{t f}\right)$ is a decreasing function of $t \geqslant 1$, and in particular is bounded, so letting $t \rightarrow \infty$ in (14) gives

$$
\lim _{t \rightarrow \infty} \int f d \mu_{t f} \geqslant \int f d \mu .
$$

Combining this with (13) we see that $\lim _{t \rightarrow \infty} \int f d \mu_{t f} \geqslant \int f d \mu$, as required.

We now show that $\mu$ is $f$-maximizing. If not then there exists $v \in \mathcal{M}$ with $\int f d v-\int f d \mu=\varepsilon>0$. Now $f$ is bounded above, so $\int f d v<\infty$. Moreover $P(f)<\infty$, so (3) and (6) imply that $h(v)<\infty$. We can then define the affine map $l_{v}: I R \rightarrow I R$ by $l_{v}(t)=h(v)+t \int f d \nu$. Now $t \mapsto p^{\prime}(t)=$ $\int f d \mu_{t f}$ is a function which increases to its limit $\int f d \mu$, so in particular

$$
\int f d \mu \geqslant \int f d \mu_{t f}=p^{\prime}(t) \text { for all } t \geqslant 1,
$$

and hence

$$
l_{\nu}^{\prime}(t)=\int f d \nu=\int f d \mu+\varepsilon \geqslant p^{\prime}(t)+\varepsilon
$$

for all $t \geqslant 1$. Therefore $l_{v}(t)>p(t)$ for all sufficiently large $t$. That is, $h(v)+\int t f d v>P(t f)$ for all sufficiently large $t$, contradicting (3). Therefore $\mu$ is $f$-maximizing. 
Note that in the case of a finite alphabet subshift of finite type $\Sigma_{A}$, the identity $\int f d \mu=\lim _{t \rightarrow \infty} \int f d \mu_{t f}$ in Theorem 1 follows immediately from the fact that $\mu$ is a weak* accumulation point of $\mu_{t f}$, since the continuous function $f$ is automatically bounded on the compact space $\Sigma_{A}$.

In the finite alphabet case some extra information is known about $\mu$ : it is of maximal entropy within the class of $f$-maximizing measures (see refs. 7-9). In the infinite alphabet case this is an open problem:

Question 1. If $\mu$ is a weak* accumulation point of $\left(\mu_{t f}\right)_{t \geqslant 1}$, is it the case that

$$
h(\mu)=\sup _{m \in \mathcal{M}_{\max }(f)} h(m) ?
$$

An approach to proving (15) is to first show that

$$
h(\mu)=\lim _{t \rightarrow \infty} h\left(\mu_{t f}\right)=\inf _{t \geqslant 1} h\left(\mu_{t f}\right)
$$

The second equality in (16) is certainly true in the infinite alphabet case, since $t \mapsto h\left(\mu_{t f}\right)$ is decreasing (as noted in the proof of Theorem 1), and bounded below. Moreover

$$
\lim _{t \rightarrow \infty} h\left(\mu_{t f}\right) \geqslant h(\mu)
$$

since $\mu_{t f}$ is the equilibrium state for $t f$, while $\mu$ is $f$-maximizing and hence $t f$-maximizing for $t \geqslant 0$, so

$$
h\left(\mu_{t f}\right)-h(\mu) \geqslant \int t f d \mu-\int t f d \mu_{t f} \geqslant 0
$$

for all $t \geqslant 1$.

We do not know, however, if equality holds in (17):

Question 2. If $\mu$ is a weak* accumulation point of $\left(\mu_{t f}\right)_{t \geqslant 1}$, is it the case that $h(\mu)=\lim _{t \rightarrow \infty} h\left(\mu_{t f}\right)$ ?

As noted above, in the finite alphabet case the answer is affirmative; this is proved by combining (17) with the well known fact (ref. 21, Thm. 8.2) that the entropy map $v \mapsto h(v)$ is upper semi-continuous on $\mathcal{M}$. 
By contrast, for infinite alphabet subshifts of finite type the entropy map is in general not upper semi-continuous. To see this, let $\Sigma$ be the full shift on $I N$, and define the probability vector $P_{n}$ by

$$
P_{n}=(1-n^{-1}, \underbrace{\left(n k_{n}\right)^{-1}, \ldots,\left(n k_{n}\right)^{-1}}_{k_{n} \text { terms }}, 0,0, \ldots),
$$

where $k_{n}=\left\lceil e^{n^{2}}\right\rceil$. Let $\mu_{n}$ be the Bernoulli measure corresponding to $P_{n}$ (so the support of $\mu_{n}$ is the full shift on the symbols $\{1, \ldots, n+1\}$ ). Its entropy (see ref. 21, p. 102) is

$$
h\left(\mu_{n}\right)=-\left(1-n^{-1}\right) \log \left(1-n^{-1}\right)+n^{-1} \log \left(n k_{n}\right)>n .
$$

In particular $h\left(\mu_{n}\right) \rightarrow \infty$ as $n \rightarrow \infty$, whereas the weak* limit of $\left(\mu_{n}\right)$ is the Dirac measure concentrated on the fixed point $(1,1, \ldots)$. This measure has zero entropy, so the entropy map is not upper semi-continuous.

Of course this absence of upper semi-continuity does not rule out an affirmative answer to Question 2. In this case Question 1 could also be answered affirmatively, by the following argument. If $h(\mu)=$ $\sup _{m \in \mathcal{M}_{\max }(f)} h(m)$ were not true then we could find $m \in \mathcal{M}_{\max }(f)$ with $h(m)-h(\mu)=\varepsilon>0$. The affirmative answer to Question 2 then gives

$$
h(m)-\lim _{t \rightarrow \infty} h\left(\mu_{t f}\right)=\varepsilon,
$$

so that

$$
h(m)-h\left(\mu_{t f}\right) \geqslant \frac{\varepsilon}{2}
$$

for sufficiently large $t \geqslant 1$. fore

But $m$ is $f$-maximizing, so $\int f d m \geqslant \int f d \mu_{t f}$ for all $t \geqslant 1$, and there-

$$
\int t f d m \geqslant \int t f d \mu_{t f}
$$

for all $t \geqslant 1$. Combining (18) and (19) gives

$$
h(m)+\int t f d m>h\left(\mu_{t f}\right)+\int t f d \mu_{t f}
$$

for $t \geqslant 1$ sufficiently large. But this is a contradiction, because $\mu_{t f}$ is an equilibrium state for the function $t f$. 


\section{ACKNOWLEDGMENTS}

The first author was partially supported by an EPSRC Advanced Research Fellowship. He acknowledges the warm hospitality of the University of North Texas, where this research was initiated. The second and third authors were supported in part by the NSF Grant No. DMS 0100078 .

\section{REFERENCES}

1. M. Aizenman and E. H. Lieb, The third law of thermodynamics and the degeneracy of the ground state for lattice systems, J. Stat. Phys. 24:279-297 (1981).

2. P. Billingsley, Convergence of Probability Measures (John Wiley \& Sons, New York, 1968).

3. R. Bowen, Equilibrium States and the Ergodic Theory of Anosov Diffeomorphisms, Springer Lecture Notes in Mathematics Vol. 470 (Berlin-Heidelberg-New York, SpringerVerlag, 1975).

4. J. Brémont, On the behaviour of Gibbs measures at temperature zero, Nonlinearity 16:419-426 (2003).

5. J. Buzzi and O. Sarig, Uniqueness of equilibrium measures for countable Markov shifts and multidimensional piecewise expanding maps, Ergod. Th. Dyn. Syst. 23:1383-1400 (2003).

6. Z. N. Coelho, Entropy and ergodicity of skew-products over subshifts of finite type and central limit asymptotics, Ph.D. Thesis (Warwick University, 1990).

7. G. Contreras, A. O. Lopes, and $\mathrm{Ph}$. Thieullen, Lyapunov minimizing measures for expanding maps of the circle, Ergod. Th. Dyn. Syst. 21:1379-1409 (2001).

8. J.-P. Conze and Y. Guivarc'h, Croissance des sommes ergodiques, manuscript, circa (1993).

9. O. Jenkinson, Geometric barycentres of invariant measures for circle maps, Ergod. Th. Dyn. Syst. 21:511-532 (2001).

10. O. Jenkinson, R. D. Mauldin and M. Urbański, Ergodic optimization for countable state subshifts of finite type, preprint.

11. A. Kerimov, Countable extreme Gibbs states in a one-dimensional model with unique ground state, J. Phys. A: Math. Gen. 31:2815-2821 (1998).

12. R. D. Mauldin and M. Urbański, Graph Directed Markov Systems: Geometry and Dynamics of Limit Sets (Cambridge University Press, 2003).

13. M. Pollicott and R. Sharp, Rates of recurrence for $\mathbb{Z}^{q}$ and $\mathbb{R}^{q}$ extensions of subshifts of finite type, J. London Math. Soc. 49:401-416 (1994).

14. C. Radin, Ordering in lattice gases at low temperature, J. Phys. A 22:317-319 (1989).

15. C. Radin, Disordered ground states of classical lattice models, Rev. Math. Phys. 3:125135 (1991).

16. C. Radin, $Z^{n}$ versus $Z$ actions for systems of finite type, Symbolic dynamics and its applications (New Haven, CT, 1991), 339-342, Contemp. Math., 135, Amer. Math. Soc., Providence, RI, 1992.

17. D. Ruelle, Thermodynamic Formalism, Encyclopaedia of Mathematics and its Applications, Vol. 5 (Addison-Wesley, 1978).

18. O. Sarig, Thermodynamic formalism for countable Markov shifts, Ergod. Th. Dyn. Syst. 19:1565-1593 (1999). 
19. O. Sarig, Characterization of existence of Gibbs measures for countable Markov shifts, Proc. Am. Math. Soc. 131:1751-1758 (2003).

20. R. Schrader, Ground states in classical lattice systems with hard core, Comm. Math. Phys. 16:247-264 (1970).

21. P. Walters, An Introduction to Ergodic Theory (Springer, 1981). 